\title{
A Network Model of Positive Resources, Temperament, Childhood Trauma, and Comorbid Symptoms for Patient with Depressive Disorders
}

\author{
Hyu Jung Huh ${ }^{1}$, Soon Young Lee ${ }^{2}$, Soo Sang Lee ${ }^{2}$, and Jeong-Ho Chae ${ }^{3 凶}$ \\ ${ }^{1}$ Department of Psychiatry, Incheon St. Mary's Hospital, The Catholic University of Korea College of Medicine, Incheon, Republic of Korea \\ ${ }^{2}$ Department of Library, Archives and Information Studies, Pusan National University, Busan, Republic of Korea \\ ${ }^{3}$ Department of Psychiatry, Seoul St. Mary's Hospital, The Catholic University of Korea College of Medicine, Seoul, Republic of Korea
}

Objective Temperament, positive resources, childhood trauma, and other clinical comorbid symptoms are related to depressive symptom severity. Here, we used network analysis to examine the interrelations between these clinical factors in patients with depressive disorders. Methods Patients with depressive disorders $(n=454)$ completed self-report questionnaires evaluating clinical symptoms, childhood trauma, temperament, and positive resources. To identify network pattern and the most central aspect, we performed network analysis and centrality analyses. First, we analyzed the network pattern in total participants. Second, we established two groups of those with severe depressive symptoms and those with mild depressive symptoms and compared their network patterns.

Results Deficient optimism and depression were the central factors in the network of total participants. In the group with severe depressive symptoms, lack of social support and childhood emotional trauma showed high centrality. Deficient social support and other positive resources played central roles in the group with mild depressive symptoms.

Conclusion Network pattern of psychological factors was different between those with mild or severe depression. Lack of positive resources is an important factor in psychological processes in both mild and severe depression. However, childhood emotional trauma may play a relatively important role in patients with severe depressive symptoms.

Psychiatry Investig 2021;18(3):214-224

Key Words Optimism, Depressive disorder, Childhood trauma, Social support.

\section{INTRODUCTION}

Depression is a prevalent mental health problem associated with substantial individual and social burdens. Many studies have been performed to explore clinical factors that influence depression symptom severity. ${ }^{1-6}$ Such clinical factors include temperament, childhood trauma, positive resources, and comorbid psychiatric symptoms. However, clinical factors of psychiatric disorder are causally mutually dependent and, thus, influence each other. ${ }^{7}$ Network analysis is a graph theory-based methodology that can be used to investigate the relationships

Received: May 18, 2020 Revised: September 20, 2020

Accepted: November 18, 2020

$\triangle$ Correspondence: Jeong-Ho Chae, MD, $\mathrm{PhD}$

Department of Psychiatry, Seoul St. Mary's Hospital, The Catholic University of Korea, College of Medicine, 222 Banpo-daero, Seocho-gu, Seoul 06591, Republic of Korea

Tel: +82-2-2258-6083, Fax: +82-2-2258-3870, E-mail: alberto@catholic.ac.kr

(c) This is an Open Access article distributed under the terms of the Creative Commons Attribution Non-Commercial License (https://creativecommons.org/licenses/by$\mathrm{nc} / 4.0$ ) which permits unrestricted non-commercial use, distribution, and reproduction in any medium, provided the original work is properly cited. between observable variables. ${ }^{8}$ This model has clinically meaningful importance for investigation of qualitative interdependent relationships of clinical factors. ${ }^{9}$ In the network model, the associations of variables can be visualized and investigated in an explorative manner on the item/symptom level, considering their complex interactions. This research method is expected to be of great help in discovering the qualitative characteristics of depression that are difficult to find in the existing quantitative research methods. Consequently, we applied network analytical methods to gain insight into the interplay of clinical factors in patients with depressive disorders.

In the following sections, we review the theoretical and empirical backgrounds of the most important connections of the behavioral activation system/behavioral inhibition system, childhood trauma, positive psychological resources, and comorbid psychiatric symptoms to further elucidate the rationale for our study in a context of depressive symptoms. 


\section{Behavioral activation system/behavioral inhibition system and depression}

There is evidence that several forms of psychopathology are characterized by specific patterns of deficits in approach- and withdrawal-behavior. In Gray's model of behavioral activation system (BAS) and behavioral inhibition system (BIS), the BAS is an approach-related, positive-incentive motivation system, while the BIS regulates sensitivity to threat and nonrewardcues. ${ }^{10}$ Although the relationship between BIS/BAS is uncertain, previous studies have reported that depression is associated with higher BIS and lower BAS activation. ${ }^{11,12}$

\section{Childhood trauma and depression}

A considerable body of studies has provided evidence that childhood trauma is associated with onset, symptom severity, and course of depression. ${ }^{13,14}$ Despite the well-established relationship between childhood trauma and adulthood mental health problems, the specific mechanism and related factors from early life trauma to later psychiatric problems are unclear. Network analysis may give insight into the complex interactions of specific types of childhood trauma, current depressive symptoms, and other clinical factors.

\section{Individual positive resources and depression}

Although many vulnerability factors including early life trauma or avoidant temperament are important subjects for studies of depression, protective factors also are critical for onset, symptom severity, and course of depression. ${ }^{15-17}$ Various positive psychological resources were investigated as protective factors of depression, including optimism, purpose in life, selfmanagement, and social support. ${ }^{18,19}$ Because protective factors are regarded as more than just absence of risk factors, interrelationships of protective factors with other vulnerable factors need to be examined. Moreover, some vulnerable factors often cannot be eliminated by intervention, and interactions of protective factors with other clinical factor are important.

\section{Depression and comorbid psychiatric symptoms (anxiety, somatization, hostility)}

Depression and anxiety are very frequent comorbid symptoms. Many empirical studies report that anxiety is a vulnerable factor for occurrence of depression. ${ }^{20-22}$ Furthermore, comorbid anxiety is related to poorer prognosis of depression and suicide. ${ }^{23}$

Both anxiety and depression are related to stressful life event, impaired cognitive processes, and negative affectivity, and they share a common biological and genetic diathesis ${ }^{24}$ Despite their common features, anxiety and depression are not identical emotional states. For example, depression, but not anxiety, is characterized by a relative deficiency of positive emotionality. In contrast, anxiety, but not depression, is characterized by hyperarousal. ${ }^{25}$ Network analysis would be helpful for elucidating the traits common and different between depression and anxiety to better understand the complex relationships with other factors.

Somatization is especially prevalent in people with depressive disorders. Several empirical studies report that depressive disorder is associated with diverse somatic symptoms. Somatization is one of the important vulnerable factors for depression. ${ }^{26}$ Furthermore, somatization is related to poorer prognosis and treatment response in depressed patients. ${ }^{27}$ Somatization can be related to other factors such as childhood trauma, comorbid anxiety, and deficiency of positive resources. ${ }^{26,28,29}$

Hostility and problematic anger have been emphasized as severity markers for the course of depression. ${ }^{30,31}$ Theoretically, in an interpersonal aspect, problematic anger is implicated as a potential social repellent that may diminish an individual's support network and contribute to feelings of isolation and worthlessness. ${ }^{32}$ Hostility is considered a cognitive component of anger that can cause individuals to be habitually suspicious of others and to behave in a defensive or aggressive manner toward others due to erroneously perceived threat. ${ }^{33}$ Several studies report that depressed patients are more likely to interpret ambiguous sentences as threatening than are non-depressed individuals. ${ }^{34}$ Therefore, hostility could be related to depressive symptom severity as well as lack of positive resources such as social support. Exploring the interrelationships among depression, hostility, and other related factors will provide meaningful insight for understanding the psychopathology of depression.

Based on these theoretical and empirical backgrounds, we model the interrelations of selected clinical factors in patients with depressive disorders. The aim of our study was twofold. The first aim was to explore the clinical factors centrally located in a network of patients with depressive disorders. Secondly, we aimed to compare and contrast the interrelationships among clinical factors between groups of patients with relatively mild depressive symptoms or relatively severe depressive symptoms. Severely depressed patients may have higher vulnerability/comorbid symptoms and lower positive resources than patients with mild depressive symptoms. Therefore, we expect that networks of patients with severe depressive symptoms and of those with mild depressive symptoms would be dissimilar in structure. With the suggested network analyses of depression-related clinical factors, we aim to provide novel insights into the architecture of empirically supported clinical factors to advance our understanding of the complex system of factors that aggravate or improve depressive symptoms. 


\section{METHODS}

\section{Participants}

We recruited patients who visited the Mood and Anxiety Disorders Unit at Seoul St. Mary's Hospital, The Catholic University of Korea, between March 2013 and May 2018. All recruited patients had a principal diagnosis of nonpsychotic depressive disorder based on the DSM-IV diagnostic criteria (major depressive disorder single episode/recurrent; mild/moderate/ severe; without psychotic features, dysthymic disorder, or depressive disorder not otherwise specified). These diagnoses were made by a psychiatrist using the Mini-International Neuropsychiatric Interview (M.I.N.I). ${ }^{35}$ Patients were eligible if they were 18-65 years old and literate in Korean. The exclusion criteria were a lifetime diagnosis of psychotic disorder, bipolar disorder, or mental retardation and any mental disorder resulting from a general medical condition. A total of 454 outpatients who met the inclusion criteria participated in this study and completed all study measures. All subjects provided written informed consent. The study protocol was approved by the Institutional Review Board of the Ethics Committee of Seoul St. Mary's Hospital at The Catholic University of Korea (KC09FZZZ0211).

\section{Measurements}

\section{Socio-demographics}

During diagnostic interviews using the M.I.N.I, patients were asked about their demographic information, including years of formal education, marital status, and employment status.

\section{Clinical symptoms (depression, anxiety, somatization, hostility)}

The Symptoms Checklist 90-revised (SCL-90-R) was used for evaluating depression, anxiety, somatization, and hostility. This checklist is a widely used questionnaire developed by Leonard R. Derogatis to determine a number of psychological symptoms. ${ }^{36}$ The SCL-90-R comprises 90 symptoms and evaluates nine symptomatic dimensions of somatization, obsessive-compulsive disorder, interpersonal sensitivity, depression, anxiety, hostility, phobic anxiety, paranoid ideation, and psychoticism.

\section{Childhood trauma}

Childhood abuse and neglect were assessed using the Childhood Trauma Questionnaire (CTQ) ${ }^{37}$ This questionnaire is a 28-item self-report inventory that assesses the following five types of potential trauma experienced by a child or teenager: emotional abuse, physical abuse, sexual abuse, emotional neglect, and physical neglect. The items are rated on a 5-point frequency scale ( $1=$ never true to $5=$ very often true). The scores were added to yield a total score for each type of trauma, ranging from 5 to 25 . Higher scores indicate greater severity.

Behavioral activation system/behavioral inhibition system We used the Behavioral Activation System/Behavioral Inhibition System scale (BIS-BAS) to assess individual differences in sensitivity of the systems. ${ }^{38}$ The BIS-BAS scale consists of 20 self-rated items using a 4-point Likert scale (1=very true for me to $4=$ very false for me). The items were combined to compose one BIS scale that addresses reactions to negative events, such as criticism. There were also three BAS scales of Drive, Fun Seeking, and Reward Responsiveness related to responses to rewarding stimuli.

\section{Positive resource test}

The Positive Resources Test is a self-report questionnaire for assessing individual positive resources in a clinical setting. ${ }^{39} \mathrm{It}$ measures 5 multi-dimensional positive resources of optimism, purpose $\&$ hope, self-control, social support, and care. The test comprises 23 items, each rated on a 5-point scale ( $1=$ very false for me to $5=$ very true for me).

\section{Data analysis}

We used network analysis to explore the relationships between clinical factors including psychiatric symptoms (depression, anxiety, hostility, somatization), temperament, childhood trauma, and positive resources. In the network model, clinical factors are defined to influence each other and to generate a psychopathological network of interacting elements. The elements in a network are called nodes, and the associations among them are called edges. For network analysis, we constructed a 2-mode matrix between participants and clinical factors. Based on Pearson's correlation as a similarity coefficient, we formed a 1-mode matrix between clinical factors and factors and transformed it into the binary matrix. If the Pearson's correlation is above \pm 0.4 , it defines 1 and if not, it defines 0 in the transformed binary matrix (Supplementary Table 1 in the online-only Data Supplement). An undirected binary network can be visualized based on a binary matrix. The nodes can be explored for their importance in the network. Network analysis allows a multivariate perspective on the complex interactions among various clinical factors. We used NetMiner 4 for network estimation, visual presentation, and centrality analysis.

\section{Network estimation and visualization}

First, we performed network analysis of the total 454 participants. We used the depression, anxiety, somatization, and hostility subscales of the SCL-90-R, the five CTQ subscales, the two BISBAS subscales, and the five POREST subscales, re- 
sulting in 16 scales as nodes in the network.

Second step, we formed two groups based on SCL-90-R depression subscore percentile. We defined the severe depressive group as SCL-90-R depression with a subscore greater than the 75 th percentile $(n=118)$ and the mild depressive group as SCL-90-R depression with a subscore less than the 25th percentile ( $\mathrm{n}=112)$. After dividing the groups, we performed network analysis in each.

\section{Centrality estimation}

For each network, we estimated the central clinical factors. We examined three types of centrality measures of the psychopathological network, degree centrality, closeness centrality, and betweenness centrality. ${ }^{40}$ Degree centrality is defined as the sum of paths connected to the focal node. A central node has many direct connections to neighboring nodes. Between- ness centrality defines the sum of the shortest paths between any two nodes in the network that involve this node. The shortest path between two nodes that are not directly connected must involve other nodes. A node with high betweenness is central in the network for information transfer and connection. Closeness centrality defines the average distance between a specific node and all other nodes in the network. A node with high closeness centrality has short paths to many other nodes and quickly reacts to changes in the network.

\section{RESULTS}

\section{Participant characteristics}

Participant demographics are presented in Table 1. The mean participant age was 35.28 ( \pm 13.16$)$ years, and $52.8 \%$ were female; $35.8 \%$ participants were married or cohabited. $73.8 \%$

Table 1. Demographic and clinical characteristics of participants

\begin{tabular}{|c|c|c|c|}
\hline Mean $(\mathrm{SD}) / \%$ & $\begin{array}{l}\text { Total participants } \\
\qquad(\mathrm{N}=454)\end{array}$ & $\begin{array}{l}\text { Participants with severe } \\
\text { depressive symptoms } \\
\qquad(\mathrm{N}=112)\end{array}$ & $\begin{array}{l}\text { Participants with mild } \\
\text { depressive symptoms } \\
(\mathrm{N}=118)\end{array}$ \\
\hline Age & $35.28(13.16)$ & $32.00(11.18)$ & $37.18(13.33)$ \\
\hline Gender (female) & 52.8 & 45.6 & 54.9 \\
\hline Educational year & $13.86(2.85)$ & $13.68(3.10)$ & $14.51(2.47)$ \\
\hline Marital status (married/cohabited) & 35.8 & 27.0 & 46.5 \\
\hline Employment status (emplolyed) & 73.8 & 66.4 & 83.3 \\
\hline \multicolumn{4}{|l|}{ Clinical symptoms (SCL-90) } \\
\hline Depression & $37.68(13.34)$ & $54.35(4.94)$ & $20.95(3.79)$ \\
\hline Anxiety & $25.71(9.58)$ & $35.25(8.05)$ & $16.53(4.80)$ \\
\hline Hostility & $12.93(5.69)$ & $17.86(5.77)$ & $8.25(2.47)$ \\
\hline Somatization & $26.93(10.73)$ & $35.36(10.96)$ & $18.26(5.42)$ \\
\hline \multicolumn{4}{|l|}{ Temperament (BIS/BAS) } \\
\hline Behavioral inhibition system & $22.87(3.51)$ & $25.20(3.11)$ & $20.23(2.86)$ \\
\hline Behavioral activation system & $34.17(6.31)$ & $33.93(6.81)$ & $34.41(6.06)$ \\
\hline \multicolumn{4}{|l|}{ Childhood trauma (K-CTQ) } \\
\hline Emotional abuse & $9.16(4.99)$ & $10.93(5.89)$ & $7.41(3.43)$ \\
\hline Emotional neglect & $22.61(8.70)$ & $23.88(9.17)$ & $19.86(7.89)$ \\
\hline Physical abuse & $16.12(2.12)$ & $17.36(1.80)$ & $14.76(1.76)$ \\
\hline Physical neglect & $8.07(3.39)$ & $8.47(3.61)$ & $7.81(3.35)$ \\
\hline Sexual abuse & $6.44(3.34)$ & $7.27(4.25)$ & $5.87(1.69)$ \\
\hline \multicolumn{4}{|l|}{ Positive resources (POREST) } \\
\hline Optimism & $19.31(5.84)$ & $14.52(4.31)$ & $24.72(4.66)$ \\
\hline Self-control & $14.03(4.33)$ & $11.51(4.07)$ & $17.34(3.59)$ \\
\hline Social support & $9.73(2.85)$ & $8.10(3.00)$ & $11.43(2.36)$ \\
\hline Purpose \& hope & $16.90(5.49)$ & $13.49(5.14)$ & $21.19(4.25)$ \\
\hline Care & $6.61(1.92)$ & $6.13(2.26)$ & $6.92(1.70)$ \\
\hline
\end{tabular}

SCL-90: Symptom Check List-90, BIS/BAS: Behavioral Inhibition System/Behavioral Activation System, K-CTQ: Korean version of Childhood Trauma Questionnaire, POREST: Positive Resource Test 
participants were employed. The mean length of education was 13.86 years $( \pm 2.85)$. The average SCL-90-R subscores of depression, anxiety, hostility and somatization score were 37.68 ( \pm 13.34$), 25.71$ ( \pm 9.58$), 12.93$ ( \pm 5.69$), 26.93$ ( \pm 10.73$)$. The mean BIS score was $22.87( \pm 3.51)$ and the mean BAS score was 34.17 ( \pm 6.31$)$. The average K-CTQ subscores of emotional abuse, emotional neglect, physical abuse, physical neglect and sexual abuse were $9.16( \pm 4.99), 22.61( \pm 8.70), 16.12( \pm 2.12)$, $8.07( \pm 3.39)$, and $6.44( \pm 3.34)$. Regarding the average subscore on the POREST, the mean scores for optimism, self-control, social support, purpose/hope and care were 19.31 ( \pm 5.84$)$, 14.03 ( \pm 4.33$), 9.73( \pm 2.85), 16.90( \pm 5.49)$, and $6.61( \pm 1.92)$, respectively.

In the group with severe depressive symptoms, the mean participant age was $32.00( \pm 11.18$ ) years, and $45.6 \%$ were female; $27.0 \%$ participants were married or cohabited. $83.3 \%$ participants were employed. The mean length of education was 13.68 years $( \pm 3.10)$. The average SCL-90-R subscores of depression, anxiety, hostility and somatization score were 54.35 ( \pm 4.94$), 35.25$ ( \pm 8.05$), 17.86$ ( \pm 5.77$), 35.36$ ( \pm 10.96$)$. The mean BIS score was $25.20( \pm 3.11)$ and the mean BAS score was 33.93 ( \pm 6.81$)$. The average K-CTQ subscores of emotional abuse, emotional neglect, physical abuse, physical neglect and sexual abuse were 10.93 ( \pm 5.89$), 23.88$ ( \pm 9.17$), 17.36( \pm 1.80)$, $8.47( \pm 3.61)$, and $7.27( \pm 4.25)$. Regarding the average subscore on the POREST, the mean scores for optimism, self-control, social support, purpose/hope and care were $14.52( \pm 4.31)$, $11.51( \pm 4.07), 8.10( \pm 3.00), 13.49( \pm 5.14)$, and $6.13( \pm 2.26)$, respectively.

In the group with mild depressive symptoms, the mean participant age was 37.18 ( \pm 13.33 ) years, and $54.9 \%$ were female; $46.5 \%$ participants were married or cohabited. $83.3 \%$ participants were employed. The mean length of education was 14.51 years $( \pm 2.47)$. The average SCL-90-R subscores of depression, anxiety, hostility and somatization score were $20.95( \pm 3.79)$, 16.53 ( \pm 4.80$), 8.25( \pm 2.47), 35.36( \pm 10.96)$. The mean BIS score was $25.20( \pm 3.11)$ and the mean BAS score was $33.93( \pm 6.81)$. The average K-CTQ subscores of emotional abuse, emotional neglect, physical abuse, physical neglect and sexual abuse were 7.41 ( \pm 3.43$), 19.86( \pm 7.89), 14.76( \pm 1.76), 7.81$ ( \pm 3.35$)$, and $5.87( \pm 1.69)$. Regarding the average subscore on the POREST, the mean scores for optimism, self-control, social support, purpose/hope and care were $24.72( \pm 4.66), 17.34( \pm 3.59), 11.43$ $( \pm 2.36), 21.19( \pm 4.25)$, and $6.92( \pm 1.70)$, respectively.

\section{Psychopathological network of total participants}

The characteristics of the psychopathological network of total participants are summarized in Table 2, and the network is visualized in Figure 1. A total of 40 edges formed among 16 nodes, and network density was 0.167 . One node formed an
Table 2. Characteristics of the psychopathological network in total participants

\begin{tabular}{lc}
\hline Number of nodes & 16 \\
Number of links & 40 \\
Number of average links & 2.5 \\
Network density & 0.167 \\
\hline
\end{tabular}

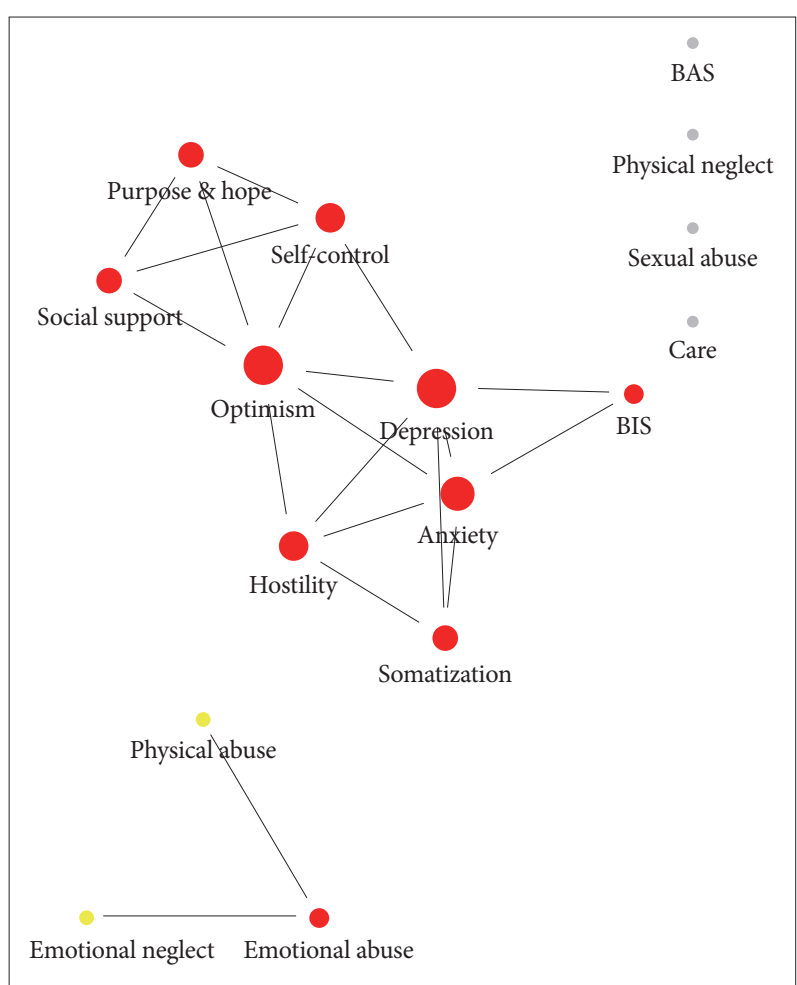

Figure 1. Psychopathological network in total participants. BAS: behavioral activation system, BIS: behavioral inhibition system.

average of 2.5 edges with another node. Of the 16 nodes, BAS, sexual abuse, and care/service were isolated nodes with no edges.

Table 3 summarizes centrality analysis of the network, revealing that optimism, depression, anxiety, self-control, and hostility had the highest degree centrality and closeness centrality. In addition, optimism, depression, anxiety, self-control, and emotional abuse had the highest betweenness centrality.

\section{Psychopathological network of participants with mild depressive symptoms}

The characteristics of the psychopathological network in participants with mild depressive symptoms are summarized in Table 4, and the network is visualized in Figure 2. A total of 26 edges formed among 16 nodes, and network density was 0.108 . Three networks and three isolated nodes appeared in the psychological network of mild depressive patients. Positive resources, four types of childhood trauma, and psychiatric symptoms formed their own respective networks. The BIS, childhood physical neglect, and sexual abuse were isolated 
Table 3. Centrality analysis of the psychopathological network in total participants

\begin{tabular}{|c|c|c|c|}
\hline & $\begin{array}{c}\text { Degree } \\
\text { centrality }\end{array}$ & $\begin{array}{l}\text { Closeness } \\
\text { centrality }\end{array}$ & $\begin{array}{c}\text { Betweenness } \\
\text { centrality } \\
\end{array}$ \\
\hline \multicolumn{4}{|l|}{ Clinical symptoms } \\
\hline Depression & 0.40 & 0.43 & 0.07 \\
\hline Anxiety & 0.33 & 0.39 & 0.03 \\
\hline Hostility & 0.27 & 0.36 & 0.01 \\
\hline Somatization & 0.20 & 0.28 & 0.00 \\
\hline \multicolumn{4}{|l|}{ Temperament } \\
\hline $\begin{array}{l}\text { Behavioral inhibition } \\
\text { system }\end{array}$ & 0.13 & 0.27 & 0.00 \\
\hline $\begin{array}{l}\text { Behavioral activation } \\
\text { system }\end{array}$ & 0.00 & 0.00 & 0.00 \\
\hline \multicolumn{4}{|l|}{ Childhood trauma } \\
\hline Emotional abuse & 0.13 & 0.13 & 0.01 \\
\hline Emotional neglect & 0.07 & 0.09 & 0.00 \\
\hline Physical abuse & 0.07 & 0.09 & 0.00 \\
\hline Physical neglect & 0.00 & 0.00 & 0.00 \\
\hline Sexual abuse & 0.00 & 0.00 & 0.00 \\
\hline \multicolumn{4}{|l|}{ Positive resource } \\
\hline Optimism & 0.40 & 0.43 & 0.08 \\
\hline Self-control & 0.27 & 0.36 & 0.02 \\
\hline Social support & 0.20 & 0.28 & 0.00 \\
\hline Purpose \& hope & 0.20 & 0.28 & 0.00 \\
\hline Care & 0.00 & 0.00 & 0.00 \\
\hline
\end{tabular}

Table 4. Characteristics of the psychopathological network in participants with mild depressive symptoms and severe depressive symptoms

\begin{tabular}{lcc}
\hline & $\begin{array}{c}\text { Participants with } \\
\text { severe depressive } \\
\text { symptoms }\end{array}$ & $\begin{array}{c}\text { Participants with } \\
\text { mild depressive } \\
\text { symptoms }\end{array}$ \\
\hline Number of nodes & 16 & 16 \\
Number of links & 22 & 26 \\
Number of average links & 1.375 & 1.625 \\
Network density & 0.092 & 0.108 \\
\hline
\end{tabular}

nodes. Compared with the network in severely depressive patients, the network density and number of edges were higher.

Tables 5, 6, and 7 summarize centrality analysis of the network, revealing that social support, purpose \& hope, self-control, and anxiety had the highest degree centrality and closeness centrality. In addition, social support, purpose \&hope, anxiety, physical abuse, and care had the highest betweenness centrality.

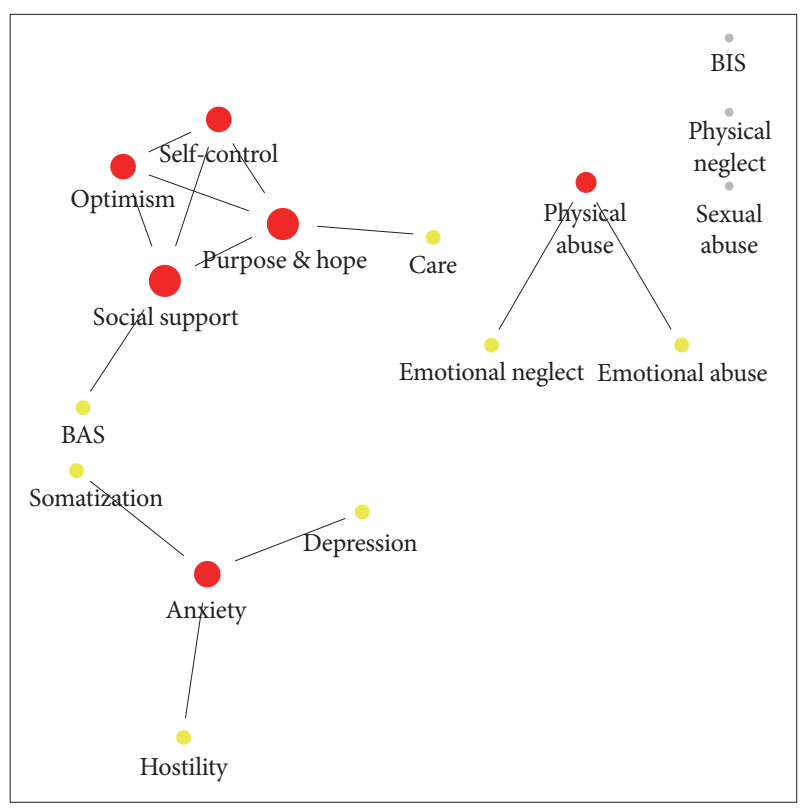

Figure 2. Psychopathological network in patients with mild depressive symptoms. BAS: behavioral activation system, BIS: behavioral inhibition system.

\section{Psychopathological network of participants with severe depressive symptoms}

The characteristics of the psychopathological network in patients with severe depressive symptoms are summarized in Table 4, and the network is visualized in Figure 3. A total of 22 edges formed among 16 nodes, and network density was 0.092 . One node formed an average of 1.375 edges with another node. Social support was connected to childhood trauma, which was different from other positive resources. Of the 16 nodes, depression, hostility, BAS, BIS, and sexual abuse were isolated, with no edges.

Tables 5, 6, and 7 summarize centrality analysis of the network, revealing that social support, self-control, emotional abuse, emotional neglect, and purpose \& hope had the highest degree centrality. Social support, emotional abuse, emotional neglect, self-control, and physical neglect had the highest closeness centrality. In addition, emotional abuse, self-control, social support, emotional neglect, and care/service had the highest betweenness centrality.

\section{DISCUSSION}

In this study, we combined temperament (BIS/BAS), childhood trauma, positive resources, comorbid anxiety, somatization, and hostility of patients with depressive disorders in a network analysis approach. We discuss the implications of the present results by analysis step in detail in the following paragraphs.

In the network of total patients, the most central node in terms of strength, closeness, and betweenness was optimism, 
Table 5. Degree centrality of the psychopathological network in participants with mild depressive symptoms and severe depressive symptoms

\begin{tabular}{lllc}
\hline & $\begin{array}{c}\text { Participants with } \\
\text { severe depressive symptoms }\end{array}$ & & $\begin{array}{c}\text { Participants with } \\
\text { mild depressive symptoms }\end{array}$ \\
\hline Social support & 0.20 & Social support & 0.27 \\
Self-control & 0.20 & Purpose \& hope & 0.27 \\
Emotional abuse & 0.20 & Self-control & 0.20 \\
Emotional neglect & 0.20 & Optimism & 0.20 \\
Purpose \& hope & 0.13 & Anxiety & 0.20 \\
Optimism & 0.13 & Physical abuse & 0.13 \\
Physical neglect & 0.13 & Care & 0.07 \\
Care & 0.07 & Emotional abuse & 0.07 \\
Physical abuse & 0.07 & Emotional neglect & 0.07 \\
Somatization & 0.07 & Behavioral activation system & 0.07 \\
Anxiety & 0.07 & Somatization & 0.07 \\
Sexual abuse & 0.00 & Hostility & 0.07 \\
Behavioral activation system & 0.00 & Depression & 0.07 \\
Behavioral inhibition system & 0.00 & Sexual abuse & Physical neglect \\
Hostility & 0.00 & Behavioral inhibition system & 0.00 \\
Depression & 0.00 & & 0.00
\end{tabular}

Table 6. Closeness centrality of the psychopathological network in participants with mild depressive symptoms and severe depressive symptoms

\begin{tabular}{lllc}
\hline & $\begin{array}{c}\text { Participants with } \\
\text { severe depressive symptoms }\end{array}$ & \multicolumn{1}{c}{$\begin{array}{c}\text { Participants with } \\
\text { mild depressive symptoms }\end{array}$} \\
\hline Social support & 0.21 & Social support & 0.28 \\
Emotional abuse & 0.21 & Purpose \& hope & 0.28 \\
Emotional neglect & 0.21 & Self-control & 0.24 \\
Self-control & 0.20 & Optimism & 0.24 \\
Physical neglect & 0.15 & Anxiety & 0.20 \\
Purpose \& hope & 0.15 & Care & 0.17 \\
Optimism & 0.15 & Behavioral activation system & 0.17 \\
Physical abuse & 0.13 & Physical abuse & 0.13 \\
Care & 0.12 & Somatization & 0.12 \\
Somatization & 0.07 & Hostility & 0.12 \\
Anxiety & 0.07 & Depression & 0.12 \\
Sexual abuse & 0.00 & Emotional abuse & 0.09 \\
Behavioral activation system & 0.00 & Emotional neglect & 0.09 \\
Behavioral inhibition system & 0.00 & Sexual abuse & 0.00 \\
Hostility & 0.00 & Physical neglect & Behavioral inhibition system \\
Depression & 0.00 & & 0.00
\end{tabular}

a kind of positive resource. In network analysis, since a node with the highest centrality has the most connections with other nodes, it can be regarded as the most important of several factors of symptom development. Therefore, the high centrality of optimism suggested that degree of optimism played a key role in the construct interaction of all scales included in the network of total participants. Lack of optimism was directly connected to depressive symptoms as well as comorbid symptoms such as anxiety and hostility. Beck's cognitive behavioral theory assumed that a negative view of oneself, the environment, and the future constitutes dysfunctional cognition of depression. Thus, negative future expectations have been con- 
HJ Huh et al.

Table 7. Betweenness centrality of the psychopathological network in participants with mild depressive symptoms and severe depressive symptoms

\begin{tabular}{lllc}
\hline & $\begin{array}{c}\text { Participants with } \\
\text { severe depressive symptoms }\end{array}$ & & $\begin{array}{c}\text { Participants with } \\
\text { mild depressive symptoms }\end{array}$ \\
\hline Emotional abuse & 0.03 & Social support & 0.04 \\
Self-control & 0.02 & Purpose \& hope & 0.04 \\
Social support & 0.01 & Anxiety & 0.03 \\
Emotional neglect & 0.01 & Physical abuse & 0.01 \\
Care & 0.00 & Care & 0.00 \\
Purpose \& hope & 0.00 & Self-control & 0.00 \\
Optimism & 0.00 & Optimism & 0.00 \\
Sexual abuse & 0.00 & Sexual abuse & 0.00 \\
Physical neglect & 0.00 & Physical neglect & 0.00 \\
Physical abuse & 0.00 & Emotional abuse & 0.00 \\
Behavioral activation system & 0.00 & Emotional neglect & 0.00 \\
Behavioral inhibition system & 0.00 & Behavioral activation system & 0.00 \\
Somatization & 0.00 & Behavioral inhibition system & 0.00 \\
Hostility & 0.00 & Somatization & 0.00 \\
Anxiety & 0.00 & Hostility & Depression \\
Depression & 0.00 & & 0.00
\end{tabular}

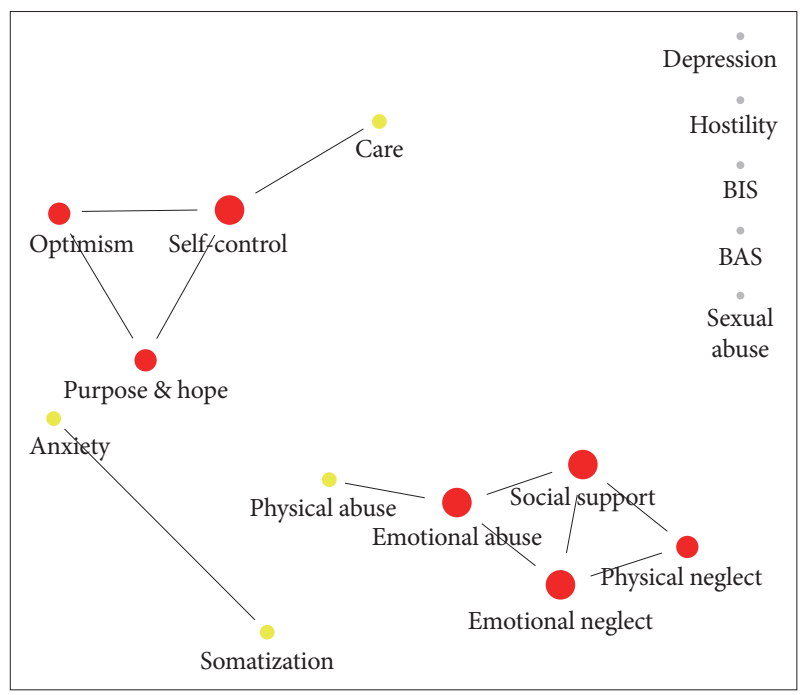

Figure 3. Psychopathological network in patients with severe depressive symptoms. BAS: behavioral activation system, BIS: behavioral inhibition system.

sidered a core feature of depression. ${ }^{41,42}$ In contrast, optimism is defined as the generalized tendency to expect the future to turn out well and is a robust predictor of psychological wellbeing. ${ }^{43,44}$ Many studies have reported that more optimistic people are less likely to develop depressive symptoms and to recover from depression more quickly. ${ }^{45,46}$ The clinical implication of this finding is that strengthening optimism could be a central treatment target for enhancing resilience from depression. By cultivating optimism, owing to its centrality, it may be assumed that depression and related comorbid symptoms will improve when optimism is increased. Other positive resources such as self-control, social support, and purpose of life/hope were also connected directly or indirectly to depression. Although not as central as optimism, positive resources are related to reduced depressive symptoms, directly or indirectly.

Comorbid anxiety and hostility also showed high centrality in the network of total participants. Both depression and anxiety were connected to BIS. An overly sensitive BIS causes negative affectivity that may contribute to vulnerability to anxiety and depression..$^{38}$ In addition, depression, anxiety, and hostility were all connected to optimism. Deficiency of optimistic thinking may also be related to negative affect/distress, contributing to anxiety, depression, and hostility. ${ }^{47,48}$ These findings suggest that different symptoms may share several common features such as vulnerable temperament and negative affectivity. Identification of modifiable transdiagnostic factors is critical to treat depressed patients with multiple comorbid symptoms.

In the network of patients with severe depressive symptoms, childhood emotional trauma and lack of social support were the central nodes in terms of strength and closeness. In betweenness centrality, childhood emotional abuse played an important role in the network of severely depressed patients. Furthermore, childhood emotional trauma was connected to deficient social support, which was dissimilar from the pattern of mildly depressed patients. These findings suggest that childhood emotional trauma holds an important position in severe depression. Previous studies were consistent with the present 
results in that childhood emotional trauma was related to depressive symptom severity. ${ }^{14,49}$ The connection between childhood trauma and social support has been supported by several empirical findings. ${ }^{50,51}$ From the perspective of cognitive-behavioral theory, various types of maladaptive schematic representations of the self, world, and future are activated by childhood emotional trauma. ${ }^{52}$ Furthermore, maladaptive schema might influence adult insecure attachment style and interpersonal dysfunction, leading to deficiency of social support. ${ }^{53}$ Therefore, strengthening social support might be difficult for patients with severe depressive symptoms because weak social support can be related to deep-rooted problems such as early traumatic experience.

Degree of social support also was the central node in terms of strength, closeness, and betweenness in the network of mildly depressed patients. In contrast with the network of patients with severe depressive symptoms, social support was connected to other positive resources such as optimism, self-control, and purpose \& hope. Inferring from the present findings, increasing social support might be interrelated with cultivation of positive resources in patients with mild depressive symptoms. Previous research has reported that individual positive psychological factors such as resilience and gratitude are associated with social support. ${ }^{54,55}$ Therefore, intervention strengthening positive psychological factors or resources might be more effective and helpful in mild depression than in severe depression.

Several limitations should be considered for the current study. First, the study used a cross-sectional design, assessing the measurements concurrently. The conclusions on mechanism of clinical factor interaction over time are hypothetical. Second, all variables, including childhood trauma, were assessed using self-report methods. It is possible that many patients have distorted mental representations of their positive resources and memories of trauma due to their depression or anxiety symptoms. Third, other factors related to childhood trauma, such as timing or length of traumas and relationships with perpetrators, were not evaluated. Despite this severe limitation, the networks produced by these analyses identify plausible interrelations between clinical factors, warranting further scrutiny.

In conclusion, lack of optimism was a central factor of patients with depressive disorders and was interrelated with depression, comorbid symptoms, and positive resources. Thus, interventions cultivating optimism may provide beneficial effect for speeding up recovery from depression and other comorbid symptoms. Comparing the interrelationships among clinical factors between the two groups of patients with relatively mild depressive symptoms or relatively severe depressive symptoms, we found different network patterns. In the network of severely depressed patients, deficiency of social support was connected to childhood emotional trauma and was a central factor. In contrast, the network of mildly depressed patients showed social support to be connected to positive resources. However, we cannot conclude a causal relationship among clinical factors based on our cross-sectional data. Study tracking changes in psychopathological network pattern before and after clinical intervention strengthening individual positive resources is necessary in the future.

\section{Supplementary Materials}

The online-only Data Supplement is available with this article at https://doi.org/10.30773/pi.2020.0187.

\section{Acknowledgments}

This study was supported by a grant of the Korea Health Technology R\&D Project through the Korea Health Industry Development Institute (KHIDI), funded by the Ministry of Health \& Welfare, Republic of Korea (grant number: HL19C0007).

\section{Conflicts of Interest}

The authors have no potential conflicts of interest to disclose.

\section{Author Contributions}

Conceptualization: Hyu Jung Huh, Jeong-Ho Chae. Data curation: Soon Young Lee, Soo Sang Lee. Formal analysis: Soon Young Lee, Soo Sang Lee. Funding acquisition: Jeong-Ho Chae. Investigation: Hyu Jung Huh, JeongHo Chae. Methodology: Soon Young Lee, Soo Sang Lee. Project administration: Jeong-Ho Chae. Resources: Jeong-Ho Chae. Software: Soon Young Lee, Soo Sang Lee. Supervision: Jeong-Ho Chae. Validation: Hyu Jung Huh, Jeong-Ho Chae. Visualization: Soon Young Lee, Soo Sang Lee. Writing_original draft: Hyu Jung Huh. Writing_-review \& editing: all authors.

\section{ORCID iDs}

Hyu Jung Huh https://orcid.org/0000-0001-8050-9189

Soon Young Lee https://orcid.org/0000-0003-4749-643X

Soo Sang Lee https://orcid.org/0000-0002-1705-0923

Jeong-Ho Chae https://orcid.org/0000-0002-6070-9324

\section{REFERENCES}

1. Lim CR, Barlas J, Ho RCM. The effects of temperament on depression according to the Schema model: a scoping review. Int J Environ Res Public Health 2018;15:1231.

2. Kampman O, Poutanen O. Can onset and recovery in depression be predicted by temperament? A systematic review and meta-analysis. J Affect Disord 2011;135:20-27.

3. Mandelli L, Petrelli C, Serretti A. The role of specific early trauma in adult depression: a meta-analysis of published literature. Childhood trauma and adult depression. Eur Psychiatry 2015;30:665-680.

4. Janiri D, Kotzalidis GD, De Chiara L, Koukopoulos AE, Aas M, Sani G. The ring of fire: childhood trauma, emotional reactivity, and mixed states in mood disorders. Psychiatr Clin North Am 2020;43:69-82.

5. Laird KT, Krause B, Funes C, Lavretsky H. Psychobiological factors of resilience and depression in late life. Transl Psychiatry 2019;9:88.

6. Graven LJ, Grant J. The impact of social support on depressive symptoms in individuals with heart failure: update and review. J Cardiovasc Nurs 2013;28:429-443.

7. Cramer AO, Waldorp LJ, van der Maas HL, Borsboom D. Comorbidity: a network perspective. Behav Brain Sci 2010;33:137-150; discussion 150-193.

8. Borsboom D, Cramer AO. Network analysis: an integrative approach to the structure of psychopathology. Annu Rev Clin Psychol 2013;9:91- 
121.

9. Fried EI. Problematic assumptions have slowed down depression research: why symptoms, not syndromes are the way forward. Front Psychol 2015;6:309.

10. Bijttebier P, Beck I, Claes L, Vandereycken W. Gray's Reinforcement Sensitivity Theory as a framework for research on personality-psychopathology associations. Clin Psychol Rev 2009;29:421-430.

11. Meyer B, Johnson SL, Winters R. Responsiveness to threat and incentive in bipolar disorder: relations of the BIS/BAS scales with symptoms. J Psychopathol Behav Assess 2001;23:133-143.

12. Johnson SL, Turner RJ, Iwata N. BIS/BAS levels and psychiatric disorder: an epidemiological study. J Psychopathol Behav Assess 2003;25: 25-36.

13. Friis RH, Wittchen HU, Pfister H, Lieb R. Life events and changes in the course of depression in young adults. Eur Psychiatry 2002;17:241253.

14. Gibb BE, Chelminski I, Zimmerman M. Childhood emotional, physical, and sexual abuse, and diagnoses of depressive and anxiety disorders in adult psychiatric outpatients. Depress Anxiety 2007;24:256263.

15. Min JA, Lee CU, Chae JH. Resilience moderates the risk of depression and anxiety symptoms on suicidal ideation in patients with depression and/or anxiety disorders. Compr Psychiatry 2015;56:103-111.

16. Min JA, Lee NB, Lee CU, Lee C, Chae JH. Low trait anxiety, high resilience, and their interaction as possible predictors for treatment response in patients with depression. J Affect Disord 2012;137:61-69.

17. Fava GA, Tomba E. Increasing psychological well-being and resilience by psychotherapeutic methods. J Pers 2009;77:1903-1934.

18. Gariepy G, Honkaniemi H, Quesnel-Vallee A. Social support and protection from depression: systematic review of current findings in Western countries. Br J Psychiatry 2016;209:284-293.

19. Li M, Jiang X, Ren Y. Mediator Effects of Positive Emotions on Social Support and Depression among Adolescents Suffering from Mobile Phone Addiction. Psychiatr Danub 2017;29:207-213.

20. Weger M, Sandi C. High anxiety trait: a vulnerable phenotype for stress-induced depression. Neurosci Biobehav Rev 2018;87:27-37.

21. Cummings CM, Caporino NE, Kendall PC. Comorbidity of anxiety and depression in children and adolescents: 20 years after. Psychol Bull 2014;140:816-845.

22. Choi KW, Kim YK, Jeon HJ. Comorbid Anxiety and Depression: Clinical and Conceptual Consideration and Transdiagnostic Treatment. Adv Exp Med Biol 2020;1191:219-235.

23. Hawton K, Casañas ICC, Haw C, Saunders K. Risk factors for suicide in individuals with depression: a systematic review. J Affect Disord 2013;147:17-28.

24. Eysenck MW, Fajkowska M. Anxiety and depression: toward overlapping and distinctive features. Cogn Emot 2018;32:1391-1400.

25. Watson D. Differentiating the mood and anxiety disorders: a quadripartite model. Annu Rev Clin Psychol 2009;5:221-247.

26. Dijkstra-Kersten SM, Sitnikova K, van Marwijk HW, Gerrits MM, van der Wouden JC, Penninx BW, et al. Somatisation as a risk factor for incident depression and anxiety. J Psychosom Res 2015;79:614-619.

27. Huijbregts KM, van Marwijk HW, de Jong FJ, Schreuders B, Beekman AT, van der Feltz-Cornelis CM. Adverse effects of multiple physical symptoms on the course of depressive and anxiety symptoms in primary care. Psychother Psychosom 2010;79:389-391.

28. Winblad NE, Changaris M, Stein PK. Effect of somatic experiencing resiliency-based trauma treatment training on quality of life and psychological health as potential markers of resilience in treating professionals. Front Neurosci 2018;12:70.

29. Gulec MY, Altintas M, Inanc L, Bezgin CH, Koca EK, Gulec H. Effects of childhood trauma on somatization in major depressive disorder: the role of alexithymia. J Affect Disord 2013;146:137-141.

30. Fava M, Hwang I, Rush AJ, Sampson N, Walters EE, Kessler RC. The importance of irritability as a symptom of major depressive disorder: results from the National Comorbidity Survey Replication. Mol Psychiatry 2010;15:856-867.

31. Judd LL, Schettler PJ, Coryell W, Akiskal HS, Fiedorowicz JG. Overt irritability/anger in unipolar major depressive episodes: past and current characteristics and implications for long-term course. JAMA Psychiatry 2013;70:1171-1180.

32. Joiner TE, Jr., Metalsky GI. A prospective test of an integrative interpersonal theory of depression: a naturalistic study of college roommates. J Pers Soc Psychol 1995;69:778-788.

33. Moreno JK, Fuhriman A, Selby MJ. Measurement of hostility, anger, and depression in depressed and nondepressed subjects. J Pers Assess 1993;61:511-523.

34. Mogg K, Bradbury KE, Bradley BP. Interpretation of ambiguous information in clinical depression. Behav Res Ther 2006;44:1411-1419.

35. Sheehan DV, Lecrubier Y, Sheehan KH, Amorim P, Janavs J, Weiller E, et al. The Mini-International Neuropsychiatric Interview (M.I.N.I.): the development and validation of a structured diagnostic psychiatric interview for DSM-IV and ICD-10. J Clin Psychiatry 1998;59(Suppl 20):22-33;quiz 34-57.

36. Derogatis LR, Rickels K, Rock AF. The SCL-90 and the MMPI: a step in the validation of a new self-report scale. Br J Psychiatry 1976;128: 280-289.

37. Bernstein D, Fink L. Childhood Trauma Questionnaire: A Retrospective Self-Report Manual. San Antonio, TX: The Psychological Corporation San Antonio; 1998.

38. Carver CS, White TL. Behavioral inhibition, behavioral activation, and affective responses to impending reward and punishment: the BIS/ BAS Scales. J Pers Soc Psychol 1994;67:319-333.

39. Huh HJ, Kim SY, Min JA, Chae JH. Development of the clinical short form positive resources test. Korean J Str Res 2018;26:77-87.

40. Hanneman RA. Riddle M. Introduction to Social Network Methods. Riverside, CA: University of California Riverside, 2005.

41. Beck AT. Cognitive Therapy of Depression. New York, NY: Guilford Press; 1979.

42. Beck JS. Cognitive Behavior Therapy: Basics and Beyond. New York, NY: Guilford Press; 2011.

43. Carver CS, Scheier MF. Dispositional optimism. Trends Cogn Sci 2014; 18:293-299.

44. Carver CS, Scheier MF, Segerstrom SC. Optimism. Clin Psychol Rev 2010;30:879-889.

45. Giltay EJ, Zitman FG, Kromhout D. Dispositional optimism and the risk of depressive symptoms during 15 years of follow-up: the Zutphen Elderly Study. J Affect Disord 2006;91:45-52.

46. Kronstrom K, Karlsson H, Nabi H, Oksanen T, Salo P, Sjosten N, et al. Optimism and pessimism as predictors of work disability with a diagnosis of depression: a prospective cohort study of onset and recovery. J Affect Disord 2011;130:294-299.

47. Kwissa-Gajewska Z, Gruszczynska E. Relationship between daily pain and affect in women with rheumatoid arthritis: lower optimism as a vulnerability factor. J Behav Med 2018;41:12-21.

48. Esteve R, Lopez-Martinez AE, Peters ML, Serrano-Ibanez ER, RuizParraga GT, Ramirez-Maestre C. Optimism, positive and negative affect, and goal adjustment strategies: their relationship to activity patterns in patients with chronic musculoskeletal pain. Pain Res Manag 2018;2018:6291719.

49. Huh HJ, Kim KH, Lee HK, Chae JH. The relationship between childhood trauma and the severity of adulthood depression and anxiety symptoms in a clinical sample: the mediating role of cognitive emotion regulation strategies. J Affect Disord 2017;213:44-50.

50. Davis JL, Petretic-Jackson PA, Ting L. Intimacy dysfunction and trauma symptomatology: long-term correlates of different types of child abuse. J Trauma Stress 2001;14:63-79.

51. DuCharme J, Koverola C, Battle P. Intimacy development: the influence of abuse and gender. J Interpers Violence 1997;12:590-599.

52. van Veen T, Wardenaar KJ, Carlier IV, Spinhoven P, Penninx BW, Zit- 
man FG. Are childhood and adult life adversities differentially associated with specific symptom dimensions of depression and anxiety? Testing the tripartite model. J Affect Disord 2013;146:238-245.

53. Platts H, Mason O, Tyson M. Early maladaptive schemas and adult attachment in a UK clinical population. Psychol Psychother 2005;78: 549-564.

54. McCanlies EC, Gu JK, Andrew ME, Burchfiel CM, Violanti JM. Resil- ience mediates the relationship between social support and post-traumatic stress symptoms in police officers. J Emerg Manag 2017;15:107116.

55. Zhang H, Zhao Q, Cao P, Ren G. Resilience and quality of life: exploring the mediator role of social support in patients with breast cancer. Med Sci Monit 2017;23:5969-5979. 
Supplementary Table 1. Correlations among the psychopathological factors in total participants

\begin{tabular}{|c|c|c|c|c|c|c|c|c|c|c|c|c|c|c|c|c|}
\hline & 1 & 2 & 3 & 4 & 5 & 6 & 7 & 8 & 9 & 10 & 11 & 12 & 13 & 14 & 15 & 16 \\
\hline 1. Depression & & 0.625 & 0.544 & 0.469 & 0.451 & 0.06 & 0.184 & 0.242 & 0.067 & 0.175 & 0.168 & -0.497 & -0.374 & -0.415 & -0.365 & -0.173 \\
\hline 2. Anxiety & & & 0.513 & 0.625 & 0.421 & 0.074 & 0.090 & 0.204 & 0.067 & 0.122 & 0.155 & -0.416 & -0.269 & -0.329 & -0.265 & -0.140 \\
\hline 3. Hostility & & & & 0.520 & 0.356 & 0.211 & 0.174 & 0.274 & 0.070 & 0.208 & 0.227 & -0.413 & -0.275 & -0.317 & -0.331 & -0.117 \\
\hline 4. Somatization & & & & & 0.307 & 0.095 & 0.101 & 0.157 & 0.063 & 0.122 & 0.175 & -0.336 & -0.198 & -0.201 & -0.195 & -0.030 \\
\hline 5. Behavioral inhibition system & & & & & & 0.152 & 0.133 & 0.150 & 0.010 & 0.050 & 0.062 & -0.350 & -0.218 & -0.327 & -0.206 & -0.131 \\
\hline 6. Behavioral activation system & & & & & & & -0.038 & 0.016 & -0.157 & 0.051 & 0.076 & 0.155 & 0.207 & 0.133 & 0.192 & 0.096 \\
\hline 7. Emotional neglect & & & & & & & & 0.490 & 0.362 & 0.361 & 0.187 & -0.288 & -0.192 & -0.242 & -0.371 & -0.096 \\
\hline 8. Emotional abuse & & & & & & & & & 0.313 & 0.545 & 0.330 & -0.214 & -0.181 & -0.267 & -0.324 & -0.054 \\
\hline 9. Physical neglect & & & & & & & & & & 0.264 & 0.170 & -0.156 & -0.070 & -0.074 & -0.312 & -0.010 \\
\hline 10. Physical abuse & & & & & & & & & & & 0.264 & -0.128 & -0.101 & -0.131 & -0.222 & -0.065 \\
\hline 11. Sexual abuse & & & & & & & & & & & & -0.077 & -0.048 & -0.100 & -0.107 & -0.023 \\
\hline 12. Optimism & & & & & & & & & & & & & 0.601 & 0.639 & 0.565 & 0.306 \\
\hline 13. Purpose \& hope & & & & & & & & & & & & & & 0.593 & 0.438 & 0.267 \\
\hline 14. Self-control & & & & & & & & & & & & & & & 0.438 & 0.309 \\
\hline 15. Social support & & & & & & & & & & & & & & & & 0.237 \\
\hline 16. Care & & & & & & & & & & & & & & & & \\
\hline
\end{tabular}

